\title{
Hybrid Modelling Approach to Study Scour Potential at Chancy-Pougny Dam Stilling Basin
}

\author{
Davide Wüthrich, Sabine Chamoun, Erik Bollaert, \\ Giovanni De Cesare and Anton J. Schleiss
}

\section{Introduction}

Chancy-Pougny Dam is a run-of-river dam located on Swiss-French border of the Rhône River downstream of Geneva. The dam is owned and operated by SFMCP (Société des Forces Motrices de Chancy-Pougny) and has a net head of about $10 \mathrm{~m}$, with a powerhouse generating some $250 \mathrm{GWh}$ of electricity per year. The main dam body contains 4 spillway gates for flood evacuation, each of them consisting of a flap gate at the crest and an orifice-type sliding gate at the base. Gates are numbered from the Swiss side towards the French side (Fig. 1). Furthermore, a fifth pass through the dam body contains a (non-equipped) ship lock on the French side. A picture of the hydropower plan is presented in Fig. 1, where both the dam and the powerhouse can be observed.

Since the dam's construction in 1920-1925, the unlined stilling basin downstream of the dam has been subjected to progressive scour of the vulnerable marls and sandstones. Hence, a hybrid modelling was performed to study the scour potential in the unprotected stilling basin and to determine adequate solutions to

D. Wüthrich · S. Chamoun · G. De Cesare $(\bowtie) \cdot$ A. J. Schleiss

EPFL, LCH, Station 18, 1015 Lausanne, Switzerland

e-mail: giovanni.decesare@epfl.ch

D. Wüthrich

e-mail: davide.wuthrich@epfl.ch

S. Chamoun

e-mail: sabine.chamoun@epfl.ch

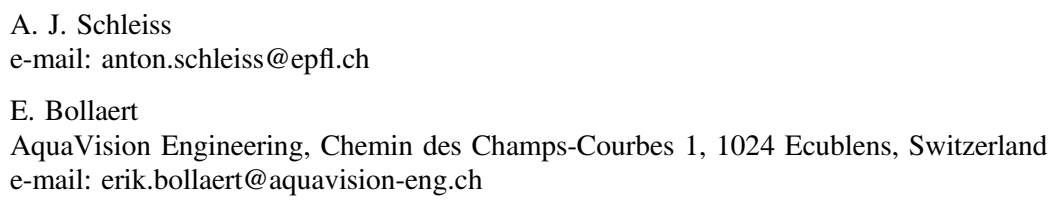




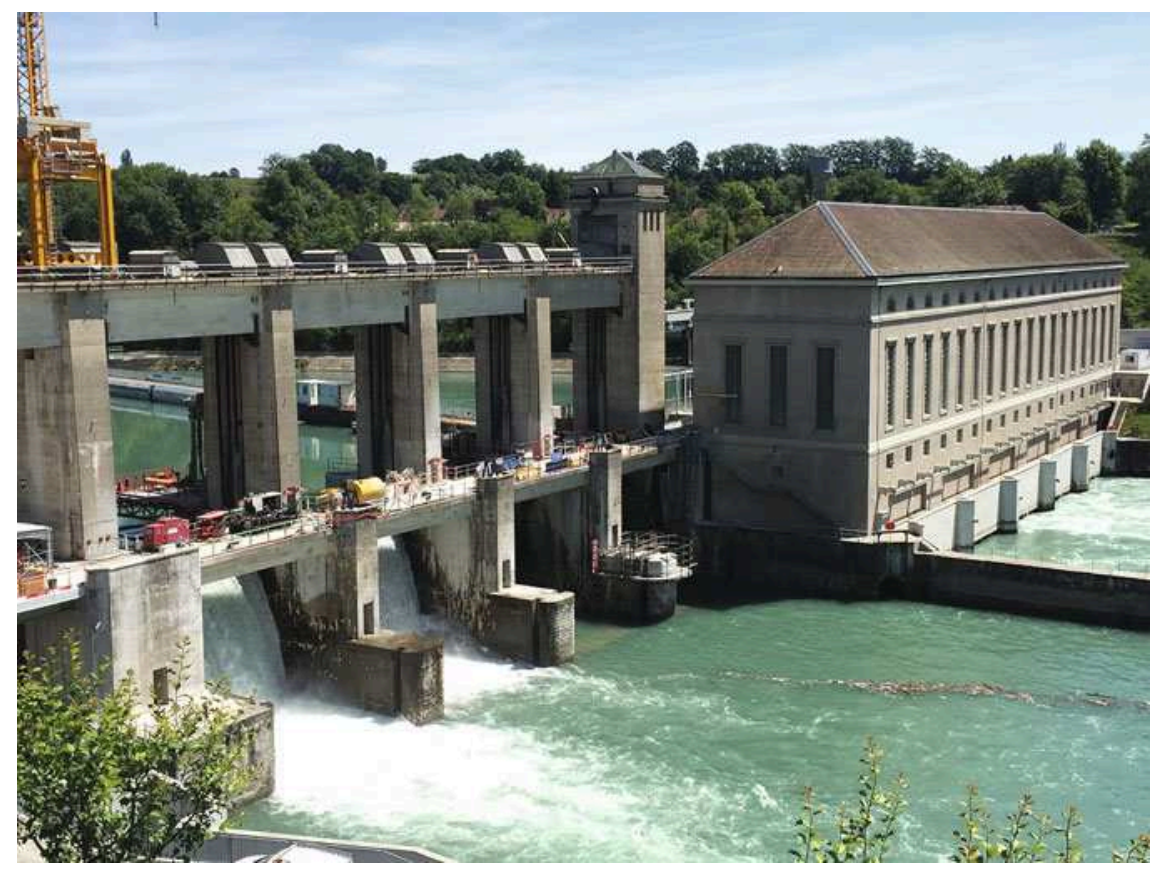

Fig. 1 Picture of the Chancy-Pougny run-of-river dam, location on the Rhône River, on the border between Switzerland and France (i.e. dam with the 4 spillway gates on the foreground, power plant on the background)

maintain future scour within acceptable levels. This hybrid modelling involves a physical model constructed at the Laboratoire de Construction Hydrauliques (LCH) at Ecole Polytechnique Fédérale de Lausanne (EPFL) and a numerical model developed at AquaVision Engineering Ltd., Ecublens, Switzerland. The physical model allows the recording of water levels, hydrodynamic pressures and flow velocities at the interface between the water and the rock. These data are then used as input into the numerical model to make predictions on future scour potential for a predefined long-term hydrology. This paper briefly presents the different solutions that were tested together with their efficiency in long-term scour protection of the stilling basin.

\section{Hybrid Modelling}

The purpose of hybrid modelling is to combine aspects of both physical and numerical modelling into one approach for a better reproduction and understanding of the involved processes. This technical paper discusses the physical modelling in 
Sect. 2.1 and the numerical modelling in Sect. 2.2. The extension of both models is presented in Fig. 2.

\subsection{Physical Model}

All experimental tests were carried out in a large-scale facility at the Laboratoire de Constructions Hydrauliques, at Ecole Polytechnique Fédérale de Lausanne (EPFL), Switzerland. The Chancy-Pougny dam and its appurtenant structures were reproduced at a 1:55 scale assuming a Froude similitude to guarantee the ratio between gravitational and inertial forces. The purpose of the physical modelling was to reproduce, in a controlled laboratory environment, the dissipation basin of the dam, the main hydraulic structures along with the upstream and downstream reaches to guarantee ideal approach conditions and reduce model effects. The components of the hydropower plant that were reproduced in the physical model are presented in Fig. 2. The topography was derived based on the bathymetry of 2006 and a linear interpolation between two sections was assumed. The riverbed was

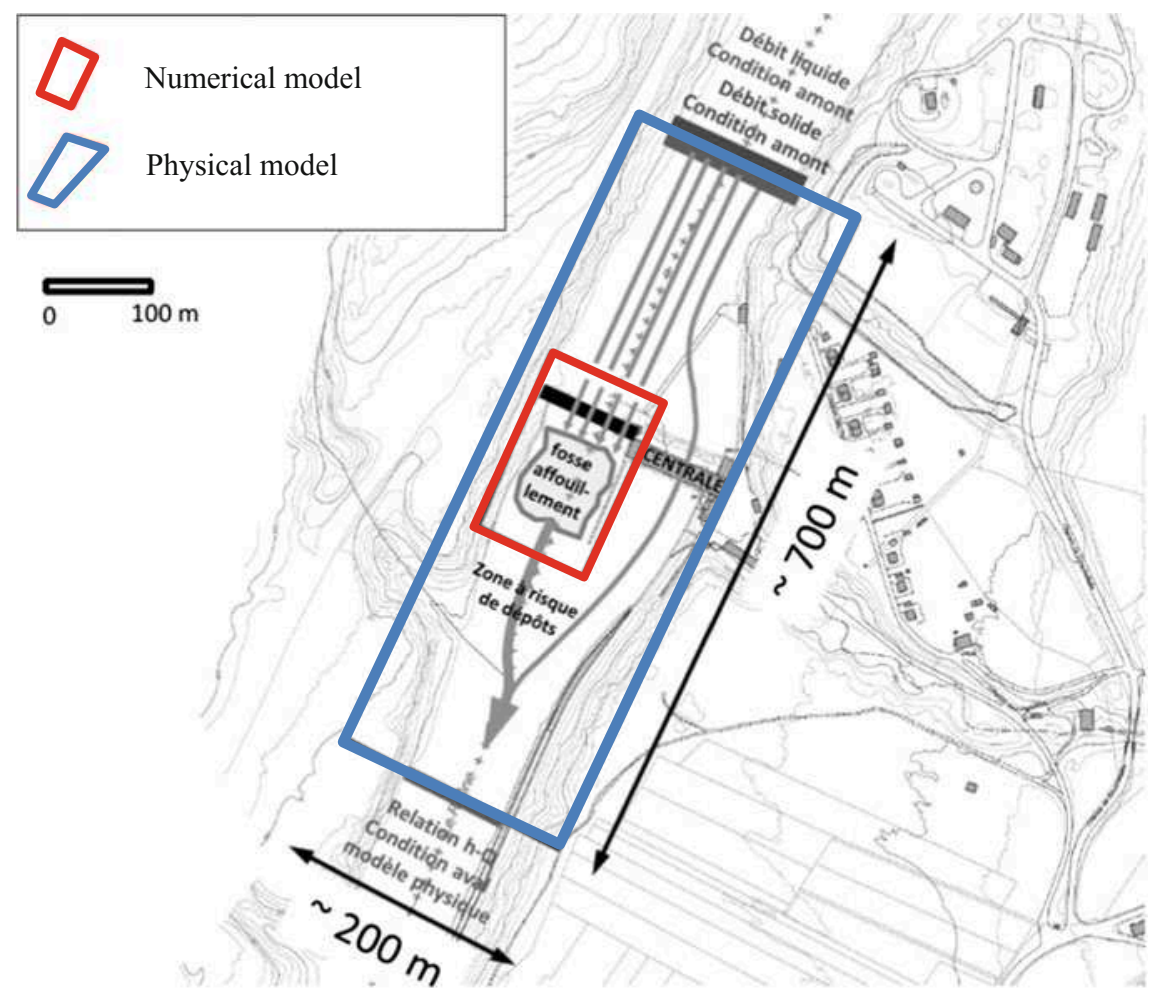

Fig. 2 Main elements reproduced in the physical and numerical models 
reproduced using a concrete surface and the hydraulic structures (dam, powerhouse, inlet grid and partition wall between dam and powerhouse) were constructed in PVC with a precision of $1 \mathrm{~mm}$. A picture of the constructed physical model is presented in Fig. 3. The water was supplied to the model through an upstream reservoir and evacuated through a gate that allowed to control the water depth in the downstream reach. A flow straightener was installed in the upstream end of the model to ensure the uniformity of the flow (Fig. 3).

The stilling basin downstream the spillway gates was constructed in a removable tank (Fig. 3b) to ease the installation of the pressure transducers and to simplify the modification of the bathymetry. Two bathymetries were considered: the original bathymetry from 1926 and an elliptical-shaped bathymetry corresponding to the ensemble of deepest scour depths obtained between 1926 and 2015. These are shown in Fig. 7a, b. However, for the present study, only the elliptical-shaped bathymetry is considered and discussed. Inside the plunge pool, the hydrodynamic pressures were recorded using 17 high-frequency pressure transducers (in two phases) providing time histories in 32 characteristic points shown in Fig. 3b. These were sampled with an acquisition frequency of $1 \mathrm{kHz}$. The water levels both inside and outside of the stilling basin were recorded using UltraSonic distance sensors (US), with an acquisition frequency of $12.5 \mathrm{~Hz}$ and a precision of $0.5 \mathrm{~mm}$.

Four operational scenarios were tested in the present study, corresponding to discharges of 550, 1080, 1575 and $2400 \mathrm{~m}^{3} / \mathrm{s}$. For all tests, no discharge through the power plant was considered, assuming that $100 \%$ of the discharge flowed through the spillway openings. The scenarios with the corresponding upstream and downstream levels and gate openings are presented in Table 1. The duration of each test was 1.5 hours at laboratory scale, corresponding to the duration of 7 hours at prototype scale.

Visual observations during the modelling showed, for all discharges, the presence of a significant flow return current along the right-hand side of the stilling basin, downstream of the ship lock. This recirculating pattern can be observed in Fig. 4a. In the same picture, an interaction between this return flow and the jet flow issuing from the spillway can also be noted. The recirculating flow, combined with

(a)

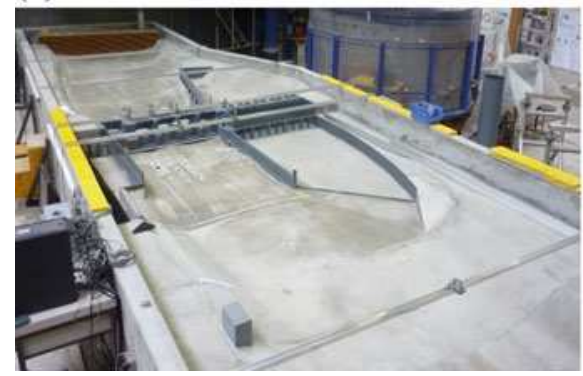

(b)

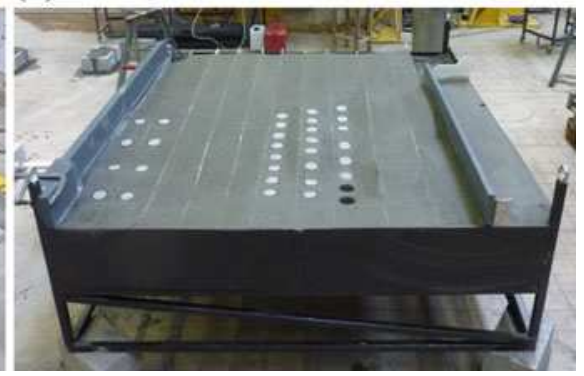

Fig. 3 Physical model constructed at LCH-EPFL. a Overview of the model; b Detailed view on the removable basin with pressure transducers 
Table 1 Four tested scenarios with the corresponding hydraulic conditions of the spillway gate

\begin{tabular}{l|l|l|l|l}
\hline $\begin{array}{l}\text { Discharge } \\
{\left[\mathrm{m}^{3} / \mathrm{s}\right]}\end{array}$ & $\begin{array}{l}\text { Return } \\
\text { period } \\
\text { [year] }\end{array}$ & $\begin{array}{l}\text { Upstream water } \\
\text { level [m a.s.l.] }\end{array}$ & $\begin{array}{l}\text { Downstream water } \\
\text { level [m a.s.1.] }\end{array}$ & $\begin{array}{l}\text { Gate opening } \\
\text { condition }\end{array}$ \\
\hline 550 & $<1$ & 347.1 & 336.4 & Overflow \\
\hline 1080 & 4 & 346.1 & 337.7 & Orifice \\
\hline 1575 & 100 & 345 & 338.4 & Orifice \\
\hline 2400 & PMF & 346.5 & 341 & $\begin{array}{l}\text { Completely } \\
\text { open }\end{array}$ \\
\hline
\end{tabular}

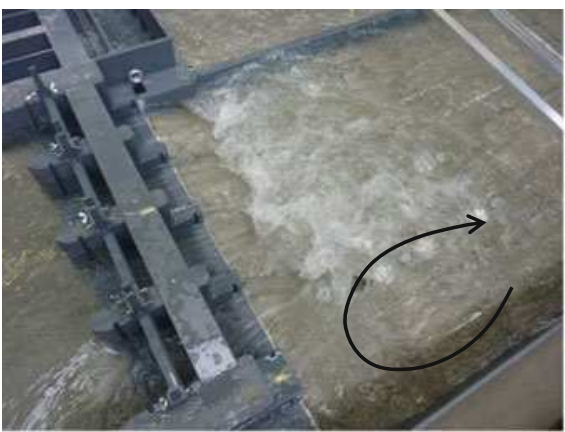

(a) flow recirculation for $\mathrm{Q}=1575 \mathrm{~m}^{3} / \mathrm{s}$

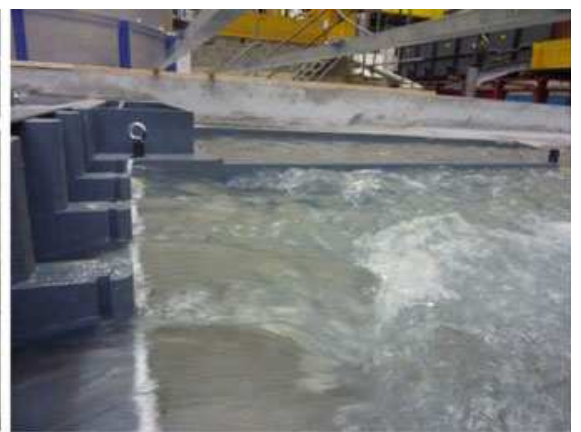

(b) vortex formation observed at gate 4 for $\mathrm{Q}=1575 \mathrm{~m}^{3} / \mathrm{s}$

Fig. 4 Flow observations: a flow recirculation; $\mathbf{b}$ formation of the turbulent vortex propagating underwater toward the rock formation

the outflow from the appurtenant gate, created a local specific discharge of almost twice the normal value, as well as a turbulent vortex impacting the rocky foundation, as shown in Fig. 4b. To validate the visual observations on the physical model, both the recirculation and the interaction with the jet were also observed on the prototype during recent extreme events. A combination of these two phenomena (return flow + vortex) was considered to be the main reason behind the locally deep erosion of the rock formation. Thus, solutions to limit this phenomenon are needed and these are presented and discussed in the following.

\subsection{Numerical Model}

The Comprehensive Scour Model comprises three methods that describe the failure of jointed rock. The Comprehensive Fracture Mechanics (CFM) method determines the ultimate scour depth by expressing instantaneous or time-dependent joint propagation due to water pressures inside the joint. The Dynamic Impulsion (DI) method describes the ejection of rock blocks from their mass due to sudden 
uplift pressures. The Quasi-Steady Impulsion Model (QSI) describes peeling off of rock blocks from their mass by quasi-steady wall jet flows. The present project has made use of the Fracture Mechanics module only. The structure of the Comprehensive Scour Model consists of three modules: the falling jet, the plunge pool and the rock mass. The latter module implements the aforementioned failure criteria. More details on equations can be found in [1].

The module of the falling jet

This module describes how the hydraulic and geometric characteristics of the jet are transformed from dam issuance down to the plunge pool water level (Fig. 5). Three parameters characterize the jet at issuance: the velocity $V_{\mathrm{i}}$, the diameter (or width) $D_{\mathrm{i}}$ (calculated using the upper nappe profile of ogee spillway flow) and the initial turbulence intensity $T_{\mathrm{u}}$, defined as the ratio of velocity fluctuations to the mean velocity. The jet trajectory is based on ballistics and air drag but calibrated by in situ observations because of the particular spillway design. The jet module computes the

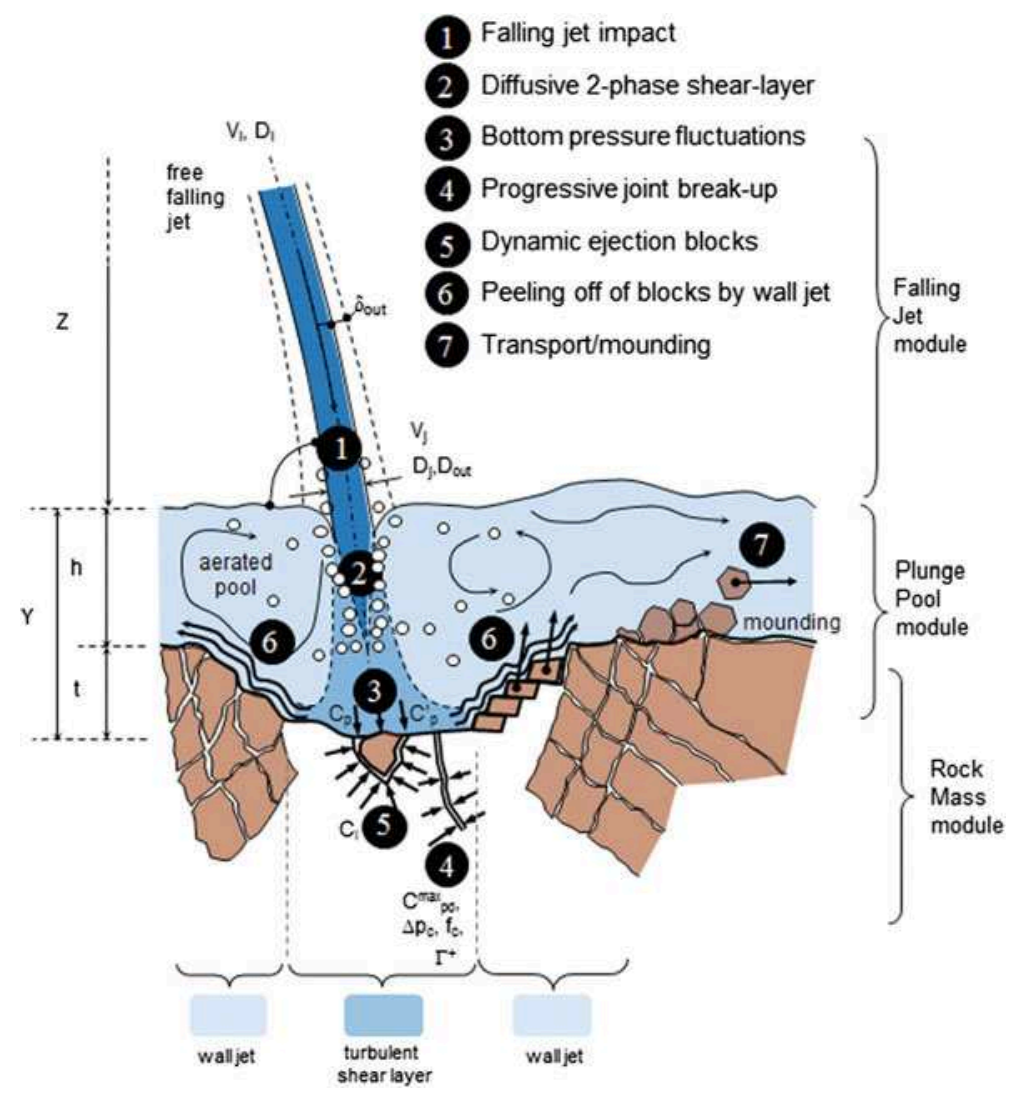

Fig. 5 Parameters of Scour model [1] 
longitudinal location of impact, the total trajectory length $L$ and the velocity and diameter at impact $V_{\mathrm{j}}$ and $D_{\mathrm{j}}$.

The module of the plunge pool

This module describes the characteristics of the jet when traversing the plunge pool and defines the water pressures at the water-rock interface. The ratio of water depth to jet diameter at impact $Y / D_{\mathrm{j}}$ is directly related to jet diffusion. The most relevant pressures are the mean dynamic pressure coefficient $C_{\mathrm{pa}}$ and the root mean square (rms) coefficient of the fluctuating dynamic pressures $C_{\mathrm{pa}}^{\prime}$, both measured at the jet centerline. These coefficients correspond to the ratio of pressure head (in $[\mathrm{m}]$ ) to incoming kinetic energy of the jet $\left(V^{2} / 2 g\right)$.

\section{The module of the rock mass}

The main parameters of this module are the following:

1. Maximum dynamic pressure coefficient $\quad C_{\mathrm{p}}^{\max }$

2. Characteristic amplitude of pressure cycles $\Delta p_{\mathrm{c}}$

3. Characteristic frequency of pressure cycles $f_{\mathrm{c}}$

4. Maximum dynamic impulsion coefficient $C_{\operatorname{maxI}}$

The first parameter is relevant to the brittle propagation of closed-end rock joints. The second and third parameters express time-dependent propagation of closed-end rock joints. The fourth parameter is used to define dynamic uplift of rock blocks formed by open-end rock joints. The maximum pressure $C_{\operatorname{maxp}}$ is obtained through multiplication of the rms pressure $C_{\mathrm{pa}}^{\prime}$ with an amplification factor $\Gamma^{+}$, and by superposition with the mean pressure $C_{\mathrm{pa}} . \Gamma^{+}$expresses the ratio of the peak value inside the rock joint to the rms value of pressures at the pool bottom and has been determined based on prototype-scaled experiments [1]. The product of $C_{\mathrm{pa}}^{\prime}$ times $\Gamma^{+}$ results in a maximum pressure, written as follows:

$$
P_{\max }[\mathrm{Pa}]=\gamma \cdot C_{\mathrm{p}}^{\max } \cdot \frac{V_{\mathrm{j}}^{2}}{2 g}=\gamma \cdot\left(C_{\mathrm{pa}}+\Gamma^{+} \cdot C_{\mathrm{pa}}^{\prime}\right) \cdot \frac{V_{\mathrm{j}}^{2}}{2 g}
$$

The characteristic amplitude of the pressure cycles, $\Delta p_{\mathrm{c}}$, is determined by the maximum and minimum pressures of the cycles. The characteristic frequency of pressure cycles $f_{\mathrm{c}}$ follows the assumption of a perfect resonator system and depends on the air concentration in the joint $\alpha_{\mathrm{i}}$ and on the length of the joint $L_{\mathrm{f}}$. These parameters are used by three methods to express rock break-up, they are discussed below.

\section{Fracture Mechanics method}

The cyclic character of pressures generated by jets makes it possible to describe joint propagation by fatigue stresses occurring at the tip of the joint. A simplified methodology [1] is called the Comprehensive Fracture Mechanics (CFM) method and applicable to any partially jointed rock. Pure tensile pressure loading inside 
rock joints is described by the stress intensity factor $K_{\mathrm{I}}$, representing the amplitude of rock mass stresses generated by water pressures at the tip of the joint. The corresponding resistance of the rock mass against joint propagation is expressed by its fracture toughness $K_{\text {Ic }}$. Joint propagation distinguishes between brittle (or instantaneous) and time-dependent joint propagation. The latter happens for a stress intensity factor equal to or higher than the fracture toughness. The latter is occurring when the maximum possible water pressure results in a stress intensity that is inferior to the material's resistance. Joints may then be propagated by fatigue. Failure by fatigue depends on the frequency and the amplitude of the load cycles. The fracture mechanics implementation of the hydrodynamic loading consists of a transformation of the water pressures in the joints into stresses in the rock. These stresses are characterized by $K_{\mathrm{I}}$ as follows:

$$
K_{\mathrm{I}}=P_{\max } \cdot F \cdot \sqrt{\pi \cdot L_{\mathrm{f}}}
$$

in which $K_{\mathrm{I}}$ is in $\mathrm{MPa} \sqrt{m}$ and $P_{\max }$ in $\mathrm{MPa}$. The boundary correction factor $F$ depends on the type of crack and on its persistency, i.e. its degree of cracking defined as $a / B$ or $b / W$ in Fig. 6. This figure presents basic configurations for partially jointed rock. The choice of the most relevant geometry depends on the type and the degree of jointing.

For practice, $F$ values of 0.5 or higher are considered to correspond to completely broken-up rock. For values of 0.1 or less, a tensile strength approach is more plausible. The fracture toughness $K_{\mathrm{Ic}}$ is related to the mineralogical type of rock and to the unconfined compressive strength UCS. Furthermore, corrections are made to account for the loading rate and the in situ stress field. The corrected fracture toughness is defined as the in situ fracture toughness $K_{\mathrm{I}, \text { ins }}$ and is based on a linear regression of available literature data. More detailed equations, as a function of the mineralogical rock composition, can be found in [2].
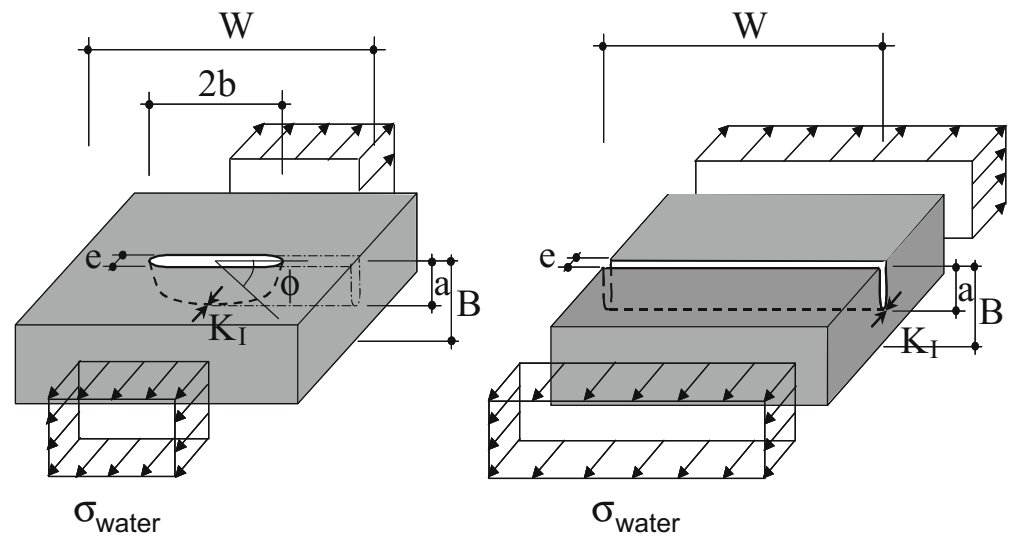

Fig. 6 Rock joint configurations of CSM model 


$$
K_{\text {Iins }, \mathrm{UCS}}=(0.008 \text { to } 0.010) \cdot \mathrm{UCS}+\left(0.054 \cdot \sigma_{\mathrm{c}}\right)+0.42
$$

in which $\sigma_{\mathrm{c}}$ represents the confinement horizontal in situ stress and UCS and $\sigma_{\mathrm{c}}$ are in MPa. Time-dependent joint propagation is expressed by an equation originally proposed to describe fatigue growth in metals:

$$
\frac{\mathrm{d} L_{\mathrm{f}}}{\mathrm{d} N}=C_{\mathrm{r}} \cdot\left(\frac{\Delta K_{\mathrm{I}}}{K_{\mathrm{Ic}}}\right)^{m_{\mathrm{r}}}
$$

in which $N$ is the number of pressure cycles. $C_{\mathrm{r}}$ and $m_{\mathrm{r}}$ are material parameters that are determined by fatigue tests and $\Delta K_{\mathrm{I}}$ is the difference between maximum and minimum stress intensity factors. To implement time-dependent joint propagation into the model, $m_{\mathrm{r}}$ and $C_{\mathrm{r}}$ have to be known. They represent the vulnerability of rock to fatigue and can be derived from available literature data.

\section{Configurations Studied}

A large number of different configurations were tested on both physical and numerical model. In the following, the three main solutions are presented and discussed. These are presented in Fig. 7 and discussed in Sects. 3.1-3.3.

\subsection{Anti-recirculation Wall}

The purpose of this configuration was to eliminate the recirculating current observed in the reference scenario, by introducing a vertical wall between gate 4 and the ship lock (Fig. 7b). A length of $30 \mathrm{~m}$ was chosen as a result of a specific 3D numerical simulation as well as systematic length variation of the wall in the physical model, proving that this value represented an optimum between effectiveness and construction costs.

For its final configuration, the wall reached a height of $341 \mathrm{~m}$ a.s.l. and no overflow was observed for any discharge presented in Table 1. Some preliminary results with lower height values were tested, but an interaction between the overflowing discharge and the jet was still observed. Visual observations proved the effectiveness of this measure and any recirculation was no longer observed for the tested discharges. Pictures of the flow with and without this constructive measure are illustrated in Fig. 8. 


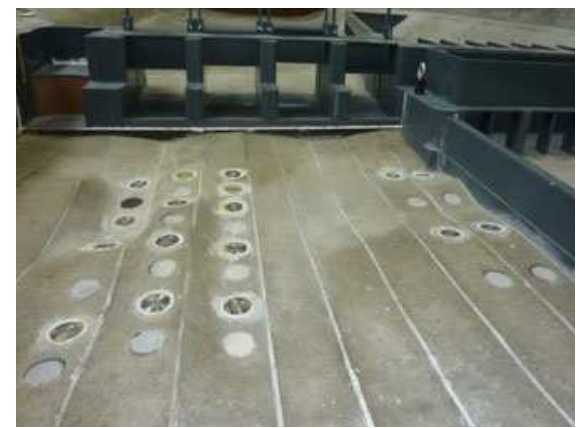

(a) reference configuration (original bathymetry)

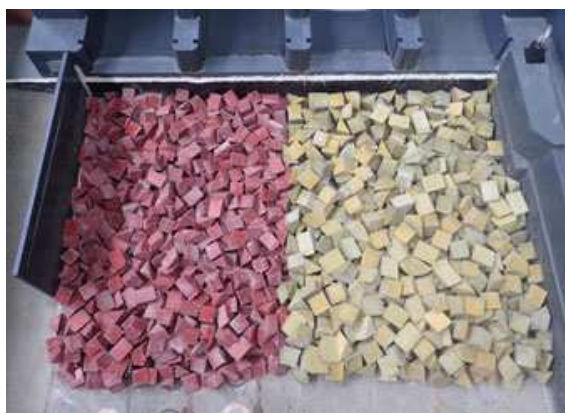

(c) half and half prisms (elliptical-shaped bathymetry)

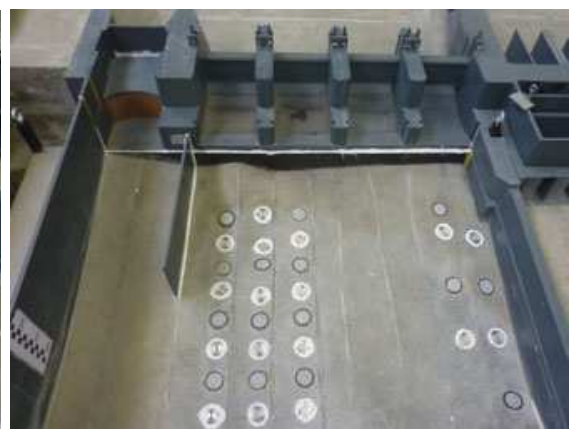

(b) anti-recirculation wall (elliptical-shaped bathymetry)

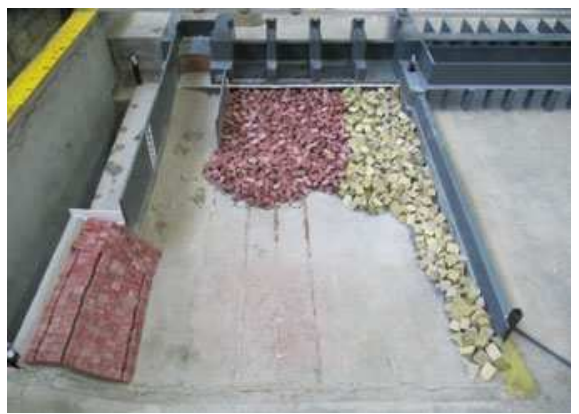

(d) retained solution (elliptical-shaped bathymetry)

Fig. 7 Various configurations tested and described in the present paper

(a)

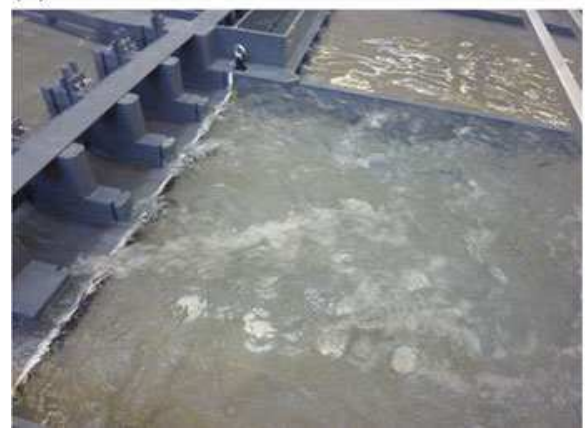

(b)

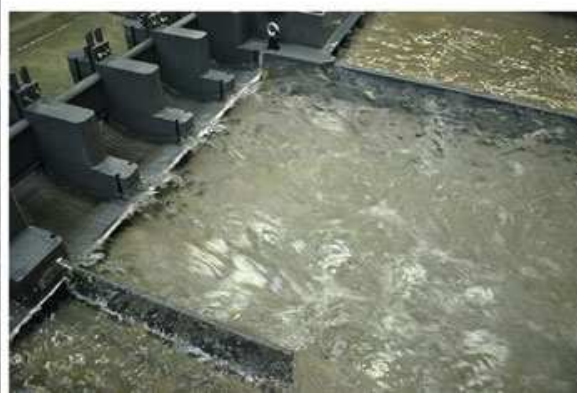

Fig. 8 Flow in the stilling basin a without and $\mathbf{b}$ with the anti-recirculation wall, for a discharge $Q=1080 \mathrm{~m}^{3} / \mathrm{s}$ 


\subsection{Concrete Prisms with Anti-recirculation Wall}

Similarly to what was previously investigated by Emami and Schleiss [3] for diversion tunnels and as bank protection in steep mountains rivers [4], a layer of concrete prisms was added to enhance the energy dissipation of the jets. The purpose of these prisms, beside a surface protection of the rock, is to deviate and destroy the jet energy before it reaches the rock formation, thus limiting the scour potential. This measure was tested in addition to the presence of the recirculating wall. Two types of prisms (diagonally divided cubes) with two sizes were tested: $1.8 \mathrm{~m}$ (red) and $2.2 \mathrm{~m}$ (yellow), corresponding to a weight of 6.8, respectively, 11.4 tons. The choice of combining both prisms, locating the smaller (red) ones on the left and the bigger (yellow) ones on the right, was derived from the outcome of previous tests, herein not discussed. For this configuration, the flow presented a fully developed hydraulic jump for all discharges, as shown in Fig. 9a. Furthermore, for all tested flow scenarios, the disposition of the prisms remained stable, as shown in Fig. 9b.

\subsection{Retained Solution}

The final solution retained for the present study corresponds to an implementation of the concrete prism configuration, presented in Sect. 3.2, by taking into account the topography of the soil in the dissipation basin. As such, the last row of prisms presented in Fig. 5d was glued to the bottom to simulate the stepped rock layers observed on the prototype. Also, an extension of the prisms along the left-hand wall separating the stilling basin from the powerhouse was adopted to guarantee the stability of the wall. Finally, this configuration was tested both with and without the anti-recirculation wall. Observations of the flow are shown in Fig. 8a. A behavior

(a)

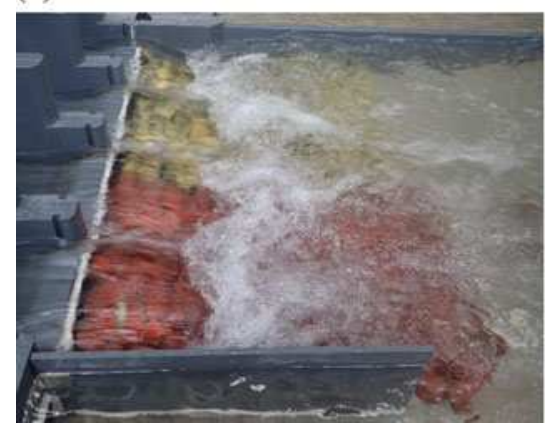

(b)

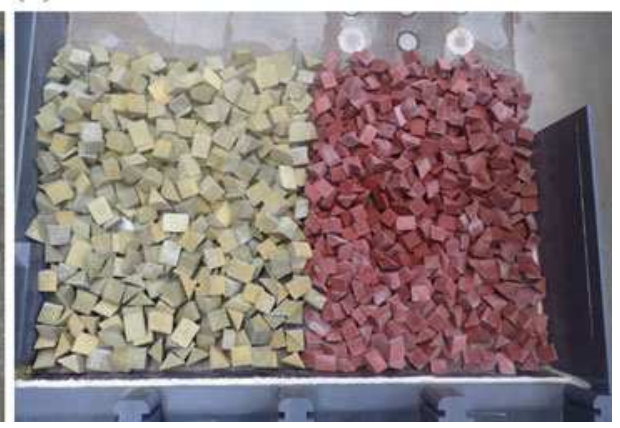

Fig. 9 Concrete prisms: a flow observations for $Q=1575 \mathrm{~m}^{3} / \mathrm{s}$ and $\mathbf{b}$ disposition of prisms after $Q=1575 \mathrm{~m}^{3} / \mathrm{s}$ 
similar to the one described for the previous configuration was noted. All hydraulic jumps were fully developed and no recirculating flow pattern was observed in the stilling basin. Furthermore, the prisms generally remained stable for all scenarios tested, with very little displacement observed. Finally, the prism configuration without the anti-recirculation wall was proved to be the best solution, with prisms that remained stable at the bottom and without any recirculating pattern (Fig. 10).

\section{Long-Term Scour Potential of the Studied Configurations}

The different configurations tested and validated on the physical model were systematically tested in the numerical model on their long-term scour potential. In a first step, the dynamic pressure fluctuations recorded on the physical model along 5 different longitudinal lines were used as input into the numerical model. Figure 11 illustrates the location of these lines and their pressure sensors, as well as the location of the deepest part of the scour formation (in dark, in between lines 3 and 5).

By using these pressure recordings as input to the fracture mechanics module, the numerical model was able to predict the scour formation as a function of time duration of overflows, for the different tested flow scenarios.

\subsection{Model Calibration}

The numerical model was calibrated by reconstituting the observed scour formation in the stilling basin during the periods 1924-1953 and 1924-2015 separately. The geology of the stilling basin was used as input into the numerical model at each

(a)

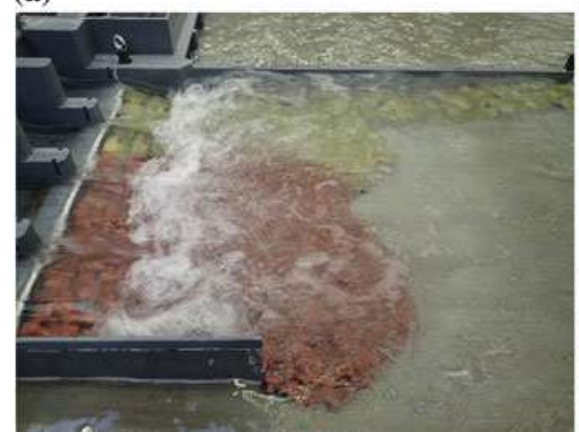

(b)

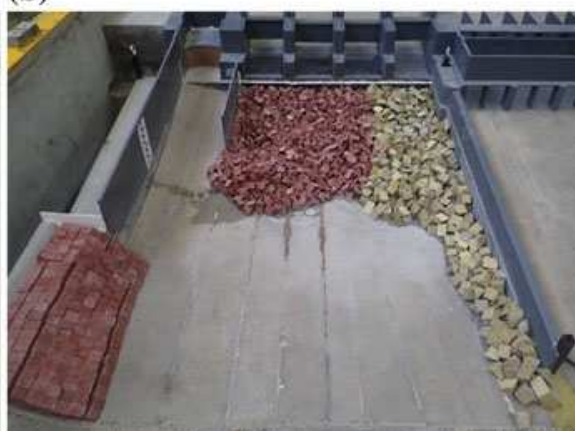

Fig. 10 Final configuration: a flow observations for $Q=1575 \mathrm{~m}^{3} / \mathrm{s}$ and b disposition of the prisms after $Q=1575 \mathrm{~m}^{3} / \mathrm{s}$ 


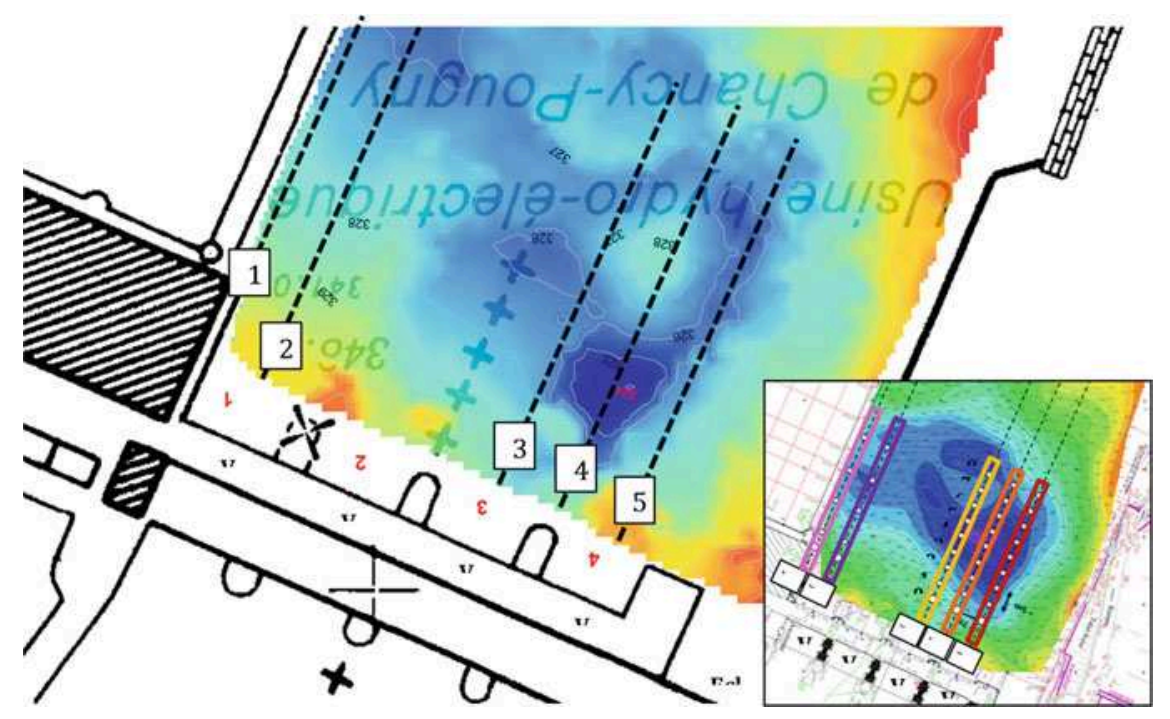

Fig. 11 Location of 5 lines of pressure recordings on the physical model

computational grid point as a function of depth of scour formation. The geology consists of scour vulnerable sedimentary layers of argillaceous limestone and sandstone, with an Unconfined Compressive Strength of only 5-20 MPa. These layers are progressively breaking up following very small pieces, and form layered steps and overhangs along their boundaries.

Figure 12 shows the calibration results for the 1924-2015 period downstream of gate No 4. By starting from the initial bottom in 1924, and passing the main floods observed during 1924-2015 through the model, a very good agreement between the computed and observed scour was obtained. Figure 12 also shows the RMS values of pressure fluctuations as recorded at different locations by the physical model.

\subsection{Scour Potential}

In a next step, the numerical model was used to predict the scour potential in the stilling basin for the next 90 years of dam operation. The hydrology was simply copied from the 1924-2015 period. This scour potential is computed for both the current situation (i.e. no scour mitigation measures) and the different configurations as described in Sect. 3 (Fig. 7), i.e. an anti-recirculation wall, a layer of concrete prisms on the bottom, and finally a combination of both wall and concrete prisms.

This is shown in Fig. 13 for the scour potential (in $\mathrm{m}$ a.s.l.) downstream of gates 3 and 4 , i.e. at the current location of deepest scour, where the turbulent vortex 


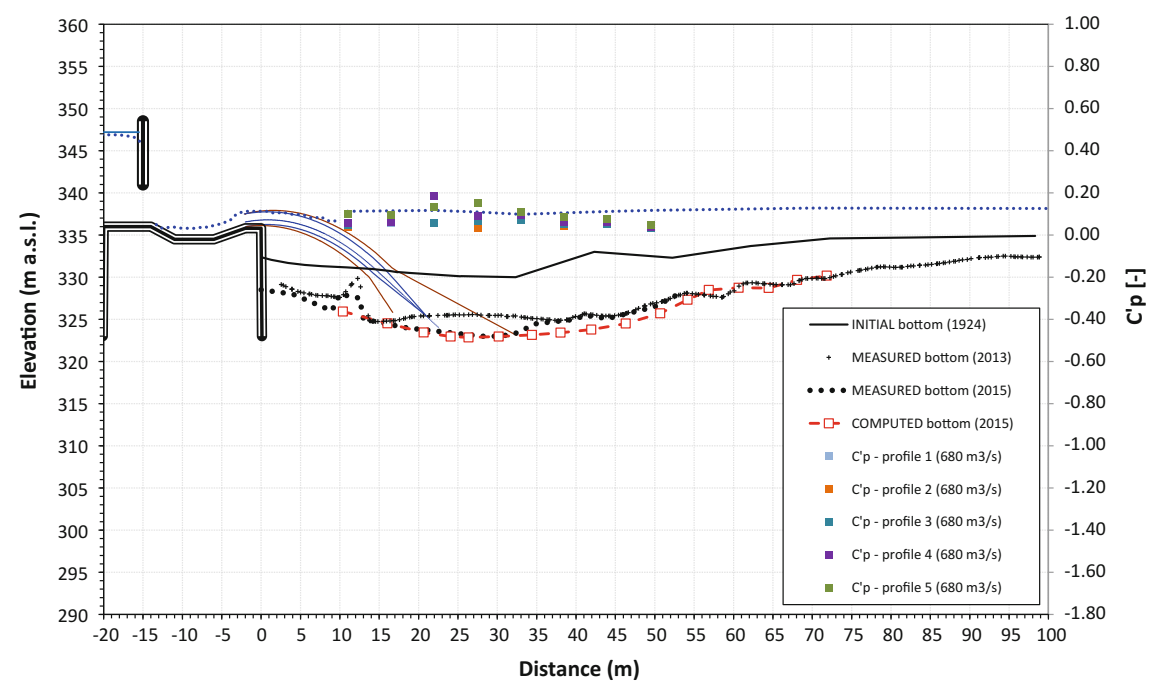

Fig. 12 Comparison of computed and observed scour formation downstream of gate No 4, for the period 1924-2015

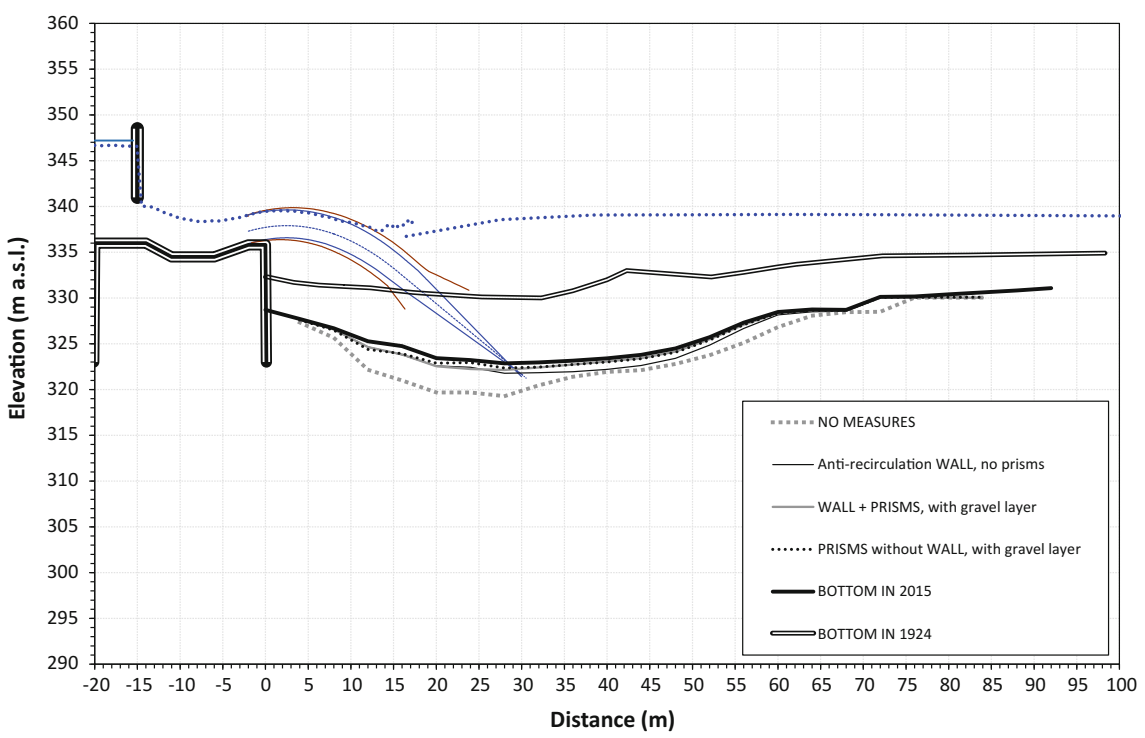

Fig. 13 Scour potential computed for the period 2015-2105: comparison of different configurations with the current situation (absolute values in $\mathrm{m}$ a.s.1.) 


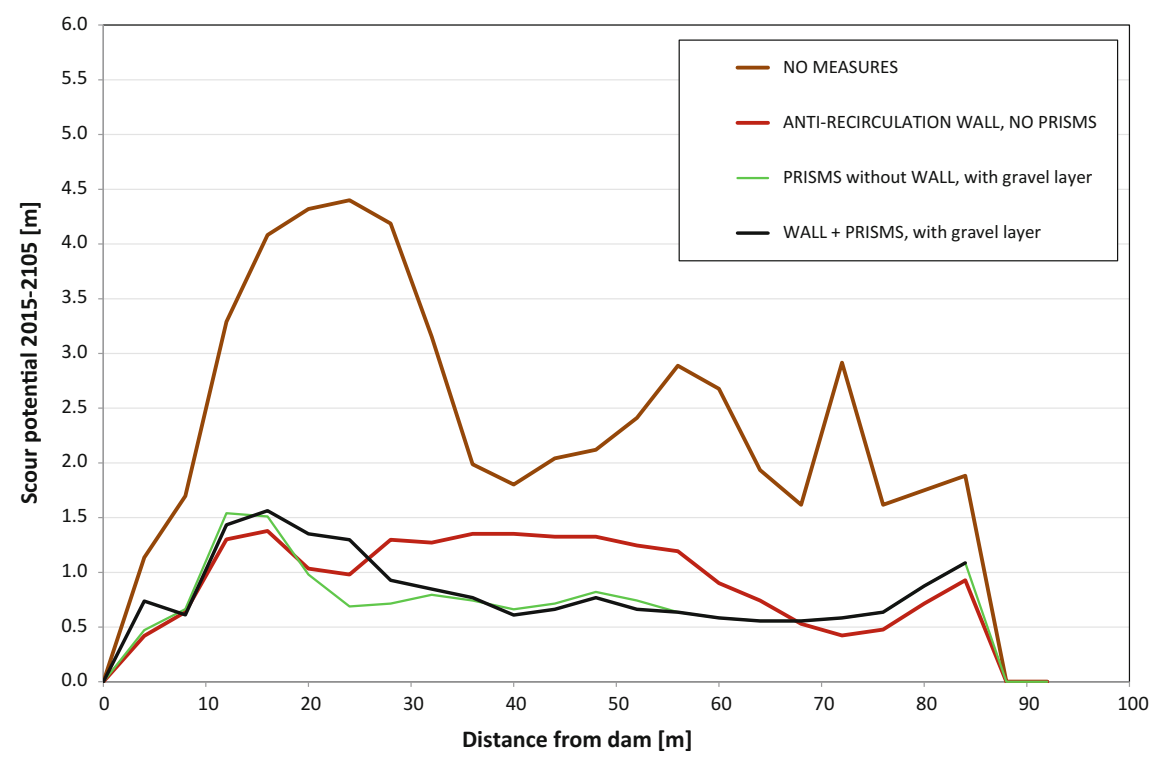

Fig. 14 Scour potential computed for the period 2015-2105: comparison of different configurations with the current situation (relative values compared to 2015 bottom)

impacts the bottom. All of the tested mitigation measures (configurations) perform significantly better than the actual situation, i.e. without mitigation measures.

The reduction in scour potential becomes more readable when expressed relative to the current bottom (2015). This is illustrated in Fig. 14 for the same tested configurations. The current plunge pool has a scour potential of almost $4.5 \mathrm{~m}$ down the current bottom level, at a location of about 15-20 m downstream of the dam. The different configurations of wall and or prisms show a future scour potential of max. 1.0-1.5 m, starting at about $15 \mathrm{~m}$ from the dam.

\section{Conclusions}

The future scour potential of the stilling basin of Chancy-Pougny Dam was assessed through hybrid modelling, combining both physical and numerical models. The physical model allowed to define the turbulent flow structure in the basin, as well as the dynamic pressure fluctuations exerted by this flow on the rocky unprotected bottom of the pool. The presence of a turbulent vortex, generated by a flow recirculation in the nonsymmetrical basin, was found to be one of the main causes of significant scour formation. The numerical model has used the pressure recordings to reconstitute the observed scour since 1924, and to determine future scour potential without any scour mitigation measures. 
Significant additional scour potential was predicted by the numerical model. As such, a series of scour mitigation measures were tested on the physical model. After visual observations of the flow structure and pressure measurements, the numerical model was used to determine the corresponding scour potential for each of the tested mitigation measures. The finally proposed mitigation measure contains a layer of concrete prisms all along the current bottom, with a side length of 1.8 and $2.2 \mathrm{~m}$, respectively.

This paper showed, once again, the importance of combining the practical results derived from a physical model with the predictions of a numerical model, allowing a better reproduction and understanding of the involved processes. Hybrid modelling is, therefore, necessary to guarantee optimal solution for better and safer hydraulic structures.

Acknowledgements The authors would like to acknowledge the SFMCP (Société des Forces Motrices de Chancy-Pougny) for commissioning the study, and all the partners involved in the study: Stucky SA (Switzerland), Norbert SA (Switzerland) and Compagnie Nationale du Rhône CNR (France).

\section{References}

1. Bollaert, E. F. R. (2004). A comprehensive model to evaluate scour formation in plunge pools. International Journal on Hydropower \& Dams, 1, 94-101.

2. Bollaert, E.F.R. (2002). Transient water pressures in joints and formation of rock scour due to high velocity jet impact (Ph.D. thesis). EPFL and Communication LCH 13.

3. Emami, S., \& Schleiss, A. J. (2006). Design of erosion protection at diversion tunnel outlets with concrete prisms. NRC-CNRC Canadian Journal of Civil Engineering, 33(1), 81-92.

4. Schleiss, A. J., Aemmer, M., Philipp, E., \& Weber, H. (1998). Erosion protection at mountain rivers with buried concrete blocks (in German). Wasser, energie, luft, Heft, 3(4), 45-52. 